\title{
Implementación y extensión del aprendizaje-servicio en las entidades de justicia global y cooperación internacional en la ciudad de Barcelona
}

\section{Maria Monzó y Judit Rifà}

Dirección de Justicia Global y Cooperación Internacional, Ayuntamiento de Barcelona

\section{Laura Rubio}

Universidad de Barcelona

\section{Resumen}

Este artículo presenta el proceso de implementación y extensión del aprendizajeservicio en el marco de las entidades que se dedican a la educación para la justicia global y la cooperación internacional en la ciudad de Barcelona. Un proceso impulsado desde el Ayuntamiento de Barcelona, conjuntamente con el Centro Promotor de Aprendizaje Servicio y el Grupo de Investigación en Educación Moral (GREM) de la Universidad de Barcelona. El artículo se inicia con el punto de partida de este proceso: la vinculación y enriquecimiento mutuo entre la educación para la justicia global y aprendizaje-servicio. Posteriormente, se apuntan las principales líneas de trabajo, acciones desarrolladas y reflexiones del propio proceso en aras de ofrecer pistas sobre la incorporación del aprendizaje-servicio desde la tarea de la administración y teniendo como núcleo las entidades sociales.

\section{Palabras clave}

Aprendizaje-servicio, entidades, justicia global, educación para el desarrollo y cooperación internacional. 


\title{
Implementation and extension of service-learning in the entities of global justice and international cooperation in the city of Barcelona
}

\begin{abstract}
This article discusses the implementation and expansion of service-learning within entities in the city of Barcelona that aim to offer education for global justice and international cooperation. This is an initiative promoted by the Barcelona City Council, together with the Service Learning Promotion Centre and the Moral Education Research Group (GREM, by its Spanish initials) of the University of Barcelona. The article begins by analysing the starting point of this process: the link between education for global justice and service-learning and the mutual enrichment they entail. The paper further discusses the main lines of work, actions developed and reflections on the process itself, in order to offer helpful information about the introduction of service-learning from an administrative perspective and keeping social entities at the core.
\end{abstract}

\section{Keywords}

Service learning, entities, global justice, education for development and international cooperation. 


\section{Introducción}

Hace un tiempo hubiera parecido extraño que un departamento de cooperación internacional de una administración pública pusiera mucho empeño en acompañar a las ONG de desarrollo, paz y derechos humanos, en incidir con mayor intención pedagógica en cambiar su realidad más próxima. Históricamente el papel de las ONG y de los gobiernos con políticas de cooperación al desarrollo ha sido dedicar esfuerzos, capacidades y presupuestos a la contribución del desarrollo en los países llamados "del sur".

En los países "del norte" las ONG tenían otro papel, el de informar, sensibilizar, y recaudar fondos, socios/as y voluntarios/as para continuar su tarea de mejora de las condiciones de vida de la población en muchos países pobres en vías de desarrollo. No era su principal misión contribuir a modificar y transformar las actitudes y prácticas de la población "del norte", ni las estructuras ni organizaciones que sostienen esta población, respecto a las situaciones de pobreza y de violación de derechos humanos en países que quedaban muy lejanos. Era una visión basada en modelos asistencialistas a través de la solidaridad de quienes dedican parte de su tiempo o dinero a "buenas causas", un modelo que no ha contribuido estructuralmente a revertir las injusticias mundiales sino que muchas veces ha permitido mantenerlas y perpetuarlas. Los datos hablan, y las desigualdades y la violación de derechos humanos de forma sistemática no disminuyen.
Poco a poco esta visión tan dicotómica, que de forma simple dividía el mundo en dos y lo hacía más comprensible para sus habitantes -el norte y el sur, los ricos y los pobres, los "desarrollándose" enfrente a "los desarrollados"-, ha quedado obsoleta, no nos sirve. Hay "nortes" en el "sur" y "sures" en el "norte", y el mundo ya no se puede explicar en blanco y negro, sino que requiere de la paleta de colores con todos sus matices. Pero sobretodo requiere que todos y todas nos situemos en un nuevo paradigma de la corresponsabilidad global. Para hacer frente a las desigualdades globales, a las relaciones de poder, económicas y comerciales, patriarcales y sociales, inherentes a un modelo de capitalismo cruel y devastador, hace falta que personas, instituciones y organizaciones se erijan sobre unos pilares basados en la justicia global, de género, ambiental, económica y garante de los derechos de las personas a desplazarse, refugiarse y migrar (Plan Director de Cooperación para la Justicia Global, 2018-2021).

Este nuevo paradigma de la justicia global puede resultar incómodo, hace que tengamos que salir de la zona de confort, y obliga a cuestionarnos nuestra forma de vida en un mundo global y globalizado, muchas veces desconocido y poco atractivo, para que pongamos esfuerzo en conocerlo. Hoy en día, es difícil ignorar que vivimos en un mundo global, de interdependencias constantes. Citando las palabras de Martha Nussbaum (2010):

"Ninguno de nosotros queda fuera de esa interdependencia. La economía global nos vincula a todos con otras

Monzó, M.; Rifà, J. y Rubio, L. (2018). Implementación y extensión del aprendizaje-servicio en las entidades de justicia global y cooperación internacional en la ciudad de Barcelona. RIDAS, Revista Iberoamericana de Aprendizaje Servicio, 6, 127-140. DOI10.1344/RIDAS2018.6.12 
personas que viven a gran distancia. Nuestras decisiones más básicas como consumidores afectan el estándar de vida de otras personas que habitan en países lejanos y que producen los artículos que usamos. Nuestra vida cotidiana presiona sobre el medio ambiente global. Por lo tanto, sería irresponsable esconder la cabeza bajo la tierra y hacer caso omiso de que todos los días nuestros actos inciden en la vida de esas otras personas. Entonces, la educación debería proporcionarnos los elementos necesarios para desenvolvernos de manera eficaz en ese diálogo multinacional, como "ciudadanos del mundo" (para usar una frase ya consagrada) y no sólo como estadounidenses, indios o europeos" ( $p$. 114).

Es importante tomar conciencia que estas relaciones interdependientes pueden llegar a generar relaciones tóxicas en un mundo que puede resultar invivible. Hoy en día las situaciones más graves de vulneración de derechos humanos y de barbaries humanas, económicas y sociales tienen el origen en causas que van más allá de las fronteras y que responden a intereses globales de los pocos (poderes masculinos) que tienen "la sartén por el mango": conflictos locales con causas globales, desplazamientos globales por causas de conflictos locales, relaciones económicas que pasan por lógicas globales, patrones de consumo que salen de toda lógica de la sostenibilidad y de la responsabilidad, patrones de violencia patriarcal basado en el modelo de la masculinidad hegemónica, empresas que violan los derechos humanos, las empresas armamentísticas o la banca armada, entre muchas otras relaciones poco saludables.

Y ahí parece que está la clave: la educación, en mayúsculas. Y es precisamente aquí cuando volvemos a la pregunta inicial de este artículo: ¿por qué un departamento de cooperación internacional de una administración pública, como es el Ayuntamiento de Barcelona, se empeña en acompañar a las organizaciones de cooperación internacional? Estas entidades (expertas en políticas de desarrollo, desigualdades globales, defensa de los derechos humanos y la paz) tienen la posibilidad de incidir en el cambio del modelo educativo hacia una pedagogía transformadora, que aproveche el currículum, la organización educativa y el sistema para repensar la educación desde la globalidad heterogénea y compleja del mundo dónde vivimos, con todas sus interdependencias y vínculos.

Las entidades sociales son agentes pedagógicos en su entorno, pueden ejercer un importante impacto educativo sobre la comunidad educativa, tanto formal, no formal como informal. Pueden ser actores de cambio destacados en este nuevo paradigma de la justicia global. Pueden contribuir a desdibujar esta línea sutil pero persistente de la división del mundo en dos mundos, y contribuir a dibujar un nuevo mapa global sin líneas divisorias. El mundo es uno y dividirlo puede facilitar su comprensión, pero paraliza el cambio y la búsqueda de la justicia global. 
Este artículo pretende explicar cómo en este marco amplio de la educación, las ONG encuentran formas de trabajar educativamente vinculadas a los grandes desafíos globales desde una mirada crítica a través de la metodología del aprendizaje-servicio.

\section{El contexto}

El año 2014 la Dirección de Justicia Global y Cooperación Internacional del Ayuntamiento de Barcelona, el Centro Promotor de Aprendizaje Servicio y el Grupo de Investigación en Educación Moral de la Universidad de Barcelona, en colaboración con el Consorcio de Educación de Barcelona y LaFede.cat, abrió una línea de trabajo para impulsar el diseño y la implementación de proyectos de aprendizaje-servicio en las entidades que trabajan en educación para la justicia global. Esta apuesta coincide, en el mismo momento, con el objetivo de las entidades de fomentar metodologías participativas, transformadoras y de mayor impacto para educar de forma crítica y potenciar las capacidades y autonomía de las personas y los colectivos.

El proceso de trabajo conjunto ha dado como resultado la puesta en marcha del Programa de Educación para la Justicia Global y aprendizaje-servicio. Por un lado, la educación para la justicia global representa un proceso de conocimiento y análisis crítico de la realidad que vincula la acción local con su dimensión global. Su finalidad es promover la conciencia crítica hacia las causas que generan desigualdades y conflictos. También contribuye al cambio de actitudes y prácticas que hacen posible una ciudadanía responsable, respetuosa y comprometida (Rubio y Lucchetti, 2016). Por otro lado, el aprendizajeservicio es una propuesta educativa que combina procesos de aprendizaje y de servicio a la comunidad en una sola actividad bien articulada, donde los participantes se forman trabajando sobre necesidades reales del entorno con el objetivo de mejorarlo (Puig et. al., 2007).

En este sentido, las principales aportaciones que supone el aprendizaje-servicio en el ámbito de la justicia global son las siguientes:

- El aprendizaje-servicio permite dar a conocer o fortalecer la misión de la entidad. Se trata de ofrecer un apoyo a las entidades para darse a conocer, lograr sus finalidades o mejorar su labor.

- Con la implicación activa, los y las jóvenes dan a conocer situaciones de injusticia social en las que trabajan las entidades y también ayudan a comprender las causas que originan estas necesidades, favoreciendo una mirada global y local sobre las mismas.

- El aprendizaje-servicio permite obtener un impacto más profundo, tanto en el ámbito social y comunitario como en el aprendizaje de los participantes, con propuestas puntuales como charlas, talleres o maletas de materiales pedagógicos.

- Las entidades, desde su ámbito de trabajo y experiencia, posibilitan la construcción de conocimiento y 
contenidos del currículum escolar de manera transversal, interdisciplinaria y crítica.

- Los proyectos de aprendizajeservicio trabajan para conectar las redes existentes y construyen nuevas redes, lo que favorece el trabajo en equipo, la implicación y la cooperación entre entidades. Así pues, se fomenta el arraigo y la potenciación del entorno para conseguir mayor impacto en la cohesión social y la participación ciudadana.

De ahí la potencia de la propuesta: la justicia global y el aprendizaje-servicio confluyen en entender la acción pedagógica como una práctica transformadora con dimensión política, orientada hacia la justicia global y a la igualdad de oportunidades, que tiene como objetivo empoderar a las personas para ejercer una ciudadanía activa y responsable, así como contribuir a provocar cambios estructurales en las organizaciones y en la propia administración pública.

En este caso, la administración pública implicada es la local, que, como tal, tiene un papel esencial y privilegiado al ser la institución más próxima a la ciudadanía y a los actores del territorio como la universidad, las organizaciones sociales o los centros educativos, entre otros. Concretamente, el programa se impulsa desde la Dirección de Justicia Global y Cooperación Internacional del Ayuntamiento de Barcelona, con el objetivo de promover políticas de educación para la justicia global y aumentar las capacidades de las entidades para que puedan convertirse en agentes educativos clave en el mapa de la ciudad y que contribuyan al "deseado" cambio y transformación social que desde diferentes ámbitos de la sociedad se reivindica, con políticas globalmente más justas, igualitarias y que promuevan romper con modelos capitalistas, patriarcales y clasistas.

A continuación se exponen las acciones que se han desarrollado con el fin de acompañar a las entidades en este proceso de acercamiento a la realidad más próxima y de poder intervenir en ella, desde el conocimiento profundo de ésta y con la colaboración de otros agentes del territorio.

\section{El Programa de Educación para la Justicia Global y aprendizaje- servicio: principios y filosofía}

La cooperación internacional y la educación para la justicia global, impulsadas tanto por las ONG como por los gobiernos locales, regionales y estatales, han vivido un largo recorrido histórico y han evolucionado a través de los años y las décadas transitando de un modelo asistencialista a otros modelos de desarrollo. Estos cambios se han experimentado a escala mundial y también en nuestro país y en la ciudad de Barcelona.

Desde la Dirección de Justicia Global y Cooperación Internacional del Ayuntamiento de Barcelona se pretende promover una acción que ponga el acento en las desigualdades y en la defensa de los derechos humanos y actúe, con una clara vocación global, sobre aquellas prácticas de poder injustas o discriminatorias que derivan en injusticias en diferentes ámbitos, 
como el económico, el ambiental y el de género. Toda esta labor se enmarca en el contexto del nuevo_Plan Director de la Dirección de Justicia Global y Cooperación Internacional del Ayuntamiento de Barcelona (2018) que empieza a desplegar una cooperación de banda ancha, es decir, la cooperación que se refiere al conjunto de las actuaciones y políticas del Gobierno más allá de las propiamente consideradas ayuda oficial al desarrollo. Se trata de iniciar un proceso hacia la coherencia de políticas.

En este contexto, las entidades que trabajan en educación para la justicia global, con un enfoque hacia la cooperación internacional, amplían su marco de trabajo. Estas entidades concretan su labor en diferentes ejes temáticos: derechos humanos, ciudadanía y gobernanza, sostenibilidad económica y social, perspectiva de género, cultura de paz, interculturalidad y medio ambiente, territorio y naturaleza (Egea, et. al, 2012 y Massip, et. al., 2018).

El cambio de paradigma hacia una educación para la justicia global parte de algunas consideraciones clave:

- Las propuestas educativas se despliegan en una ciudad educadora donde todos los agentes de la ciudad tienen un papel activo en relación con el cambio a favor de la justicia global.

- El territorio y el contexto también tienen una destacada importancia en este nuevo paradigma. Las acciones que se impulsan pasan en un barrio concreto de la ciudad con un tejido de entidades propio, con unos proyectos de barrio determinados y con una población específica.

- Finalmente, se busca incidir sobre las causas estructurales de las injusticias. La voluntad de este nuevo paradigma es contribuir a tomar conciencia respecto de las numerosas interdependencias entre el mundo local y el mundo global poniendo énfasis en los mecanismos que generan desigualdades.

En la propuesta que se desarrolla a continuación, y que se sitúa en este nuevo paradigma de la educación para la justicia global, las entidades redimensionan su labor educativa y de sensibilización con los y las jóvenes. Ahora las entidades se acercan a sus formas de actuar, al currículum y, en general, a los centros educativos, desde una nueva perspectiva. De este modo, descubren una forma de trabajar compartida en la que, junto con las instituciones educativas, se reconoce su labor como agentes educativos con un proyecto conjunto.

El Programa de Educación para la Justicia Global y aprendizaje-servicio pretende favorecer la incorporación de esta propuesta en la acción educativa de las entidades de la ciudad de Barcelona. En este sentido, se trabaja directamente con las entidades para promover este tipo de proyectos, así como también para velar por la construcción de una fuerte red local de colaboraciones que permita dar sentido y cobertura al trabajo coordinado entre los centros educativos y otros agentes del territorio. En definitiva, se trata de

Monzó, M.; Rifà, J. y Rubio, L. (2018). Implementación y extensión del aprendizaje-servicio en las entidades de justicia global y cooperación internacional en la ciudad de Barcelona. RIDAS, Revista Iberoamericana de Aprendizaje Servicio, 6, 127-140. DOI10.1344/RIDAS2018.6.12 
incorporar en las entidades de educación para la justicia global propuestas pedagógicamente ricas, de carácter transformador y con mayor impacto. Su labor formativa ha de permitir cada vez más la participación y la implicación social de los y las jóvenes en su entorno, reforzar su conciencia crítica hacia las desigualdades y contribuir al cambio de actitudes y prácticas a favor de la justicia global. En este marco, el aprendizaje-servicio, así como también la teoría del cambioi, se han convertido en elementos clave para lograr progresivamente una nueva forma de pensar y de hacer en la educación para la justicia global.

\section{Acciones para la introducción y la implementación del aprendizaje- servicio}

La línea de trabajo piloto que se inició en 2013 se ha convertido hoy en un programa de impulso del aprendizajeservicio desde las entidades de justicia global que se concreta en diferentes acciones que se organizan a través de diversas líneas de trabajo.

\begin{tabular}{|l|l|}
\hline $\begin{array}{l}\text { Líneas de } \\
\text { trabajo }\end{array}$ & Acciones \\
\hline $\begin{array}{l}\text { Dotación } \\
\text { de } \\
\text { recursos }\end{array}$ & $\begin{array}{l}\text { Línea de subvenciones } \\
\text { piloto para el desarrollo } \\
\text { de proyectos }\end{array}$ \\
$\begin{array}{l}\text { Apertura de una } \\
\text { convocatoria de } \\
\text { subvenciones específica } \\
\text { para proyectos de } \\
\text { aprendizaje-servicio }\end{array}$ \\
$\begin{array}{l}\text { Aportación en formato de } \\
\text { subvenciones a entidades }\end{array}$ \\
\hline
\end{tabular}

\begin{tabular}{|c|c|}
\hline & $\begin{array}{l}\text { que despliegan su } \\
\text { actuación en los distritos }\end{array}$ \\
\hline $\begin{array}{l}\text { Forma- } \\
\text { ción y } \\
\text { acompa- } \\
\text { ñamiento }\end{array}$ & $\begin{array}{l}\text { Realización de un curso } \\
\text { de introducción al } \\
\text { aprendizaje-servicio para } \\
\text { entidades } \\
\text { Realización de seminarios } \\
\text { para entidades y } \\
\text { personas expertas } \\
\text { Asesoramiento a } \\
\text { entidades y proyectos }\end{array}$ \\
\hline $\begin{array}{l}\text { Creación } \\
\text { de redes y } \\
\text { arraigo al } \\
\text { territorio }\end{array}$ & $\begin{array}{l}\text { Creación de red a través } \\
\text { de los proyectos de } \\
\text { aprendizaje-servicio } \\
\text { Trabajo conjunto como } \\
\text { procedimiento para } \\
\text { desarrollar el programa } \\
\text { Participación y formación } \\
\text { de redes para la } \\
\text { extensión de la propuesta }\end{array}$ \\
\hline $\begin{array}{l}\text { Recono- } \\
\text { cimiento y } \\
\text { comuni- } \\
\text { cación }\end{array}$ & $\begin{array}{l}\text { Elaboración de una guía } \\
\text { de trabajo } \\
\text { Grabación de vídeos de } \\
\text { experiencias } \\
\text { Elaboración de un espacio } \\
\text { web y mapa de buenas } \\
\text { prácticas } \\
\text { Jornada "Educación para } \\
\text { la justicia global" } \\
\text { Realización de jornadas } \\
\text { de reconocimiento e } \\
\text { intercambio de buenas } \\
\text { prácticas en los distritos } \\
\text { Evaluación del impacto } \\
\text { del proceso } \\
\text { Redacción de artículos de } \\
\text { difusión y presentación }\end{array}$ \\
\hline
\end{tabular}


en congresos

Tabla 1. Acciones realizadas hasta ahora por cada una de las líneas de trabajo del programa.

Por un lado, destaca la dotación de recursos a las entidades para garantizar la viabilidad y el desarrollo de los proyectos de aprendizaje a través de la convocatoria de subvenciones. En 2014 se ayudaron, con una pequeña cantidad económica y de forma piloto, tres proyectos fruto del primer curso de formación. La subvención permitió a estos proyectos un primer impulso para llevar a cabo experiencias. Este apoyo fue acompañado de asesoramiento específico para llevar a cabo el proyecto de aprendizaje-servicio propuesto por la entidad. A partir de aquí, se apostó por incorporar una línea específica de proyectos de aprendizaje-servicio en la convocatoria ordinaria de subvenciones de la Dirección de Justicia Global y Cooperación Internacional del Ayuntamiento de Barcelona. De esta forma se consolida el compromiso de la dirección con los proyectos colaborativos entre ONG y entidades educativas a través del aprendizajeservicio. Desde el año 2014 se han subvencionado 64 proyectos en la ciudad con un presupuesto total otorgado de 834.781,17€.

Por otra parte, el despliegue de la justicia global en la ciudad tiene un fuerte componente territorial. Esto implica la creación de redes e implementación en el territorio a través del aprendizaje-servicio, del trabajo conjunto como procedimiento para desarrollar el programa y de la participación y formación de redes para la extensión de la propuesta. Las acciones se desarrollan en un territorio, barrio o distrito determinado y que normalmente su definición e implementación viene condicionada por los elementos que conforman el territorio, tanto a nivel de actores como de impulso de determinadas líneas de acción política, o bien las propias dinámicas comunitarias que se dan en estos territorios (redes, plataformas, movimientos, planes comunitarios, etc.). En consonancia con este despliegue territorial, se han realizado aportaciones en formato de subvenciones y asesoramiento a entidades que realizan su actuación en los distritos. Normalmente se trata de entidades con poca estructura y muy basadas en el voluntariado que trabajan en los distritos y que se proponen realizar proyectos de aprendizaje-servicio. En este caso, las ayudas se vehiculan a través de los distritos de la ciudad.

También, un punto crucial del programa tiene que ver con la formación y acompañamiento. Se apuesta por la apertura de espacios formativos y de intercambio entre las entidades como cursos, seminarios y asesoramientos individuales. Desde el 2013 se realizan espacios que integran la formación, intercambio de experiencias, así como momentos de reflexión y creación de aprendizaje compartido. Han pasado por los seminarios una media de 25 entidades de cooperación internacional y justicia global de la ciudad durante cada curso desde el inicio del programa. 
Las formaciones vienen acompañadas de asesoramientos que dan respuesta a cuestiones prácticas en la puesta en marcha de proyectos, necesidades compartidas y construcción de experiencias. Durante el curso 20172018 los asesoramientos a entidades de ciudad se han reconvertido en una bolsa de horas de acompañamiento que pueden usar las diferentes entidades según requiera su proyecto. Los encuentros de seguimiento a nivel grupal han sido clave a la hora de identificar y atender necesidades específicas del proceso de diseño, implementación y evaluación del propio proyecto.

Por último, a nivel de reconocimiento $y$ comunicación, se ha sistematizado y difundido todo lo que ha generado el programa como buenas prácticas en proyectos de aprendizaje-servicio (elaboración de guías, jornadas de reconocimiento, artículos, evaluación del proceso, etc.).

\section{Las claves del éxito y retos para la extensión del aprendizaje- servicio}

El programa ha sabido aprovechar un momento en que las entidades se replantean sus prácticas y reflexionan sobre un cambio de paradigma buscando nuevas experiencias educativas que permitan un cambio de conciencia y hábitos en nuestras realidades más próximas que se traslade en situaciones más justas en otros contextos. Por ello se considera importante trabajar desde Barcelona deconstruyendo ciertas actitudes poco favorecedoras de la convivencia: el racismo, la violencia de género, la sobreexplotación de recursos naturales, entre otras; tanto para mejorar en la construcción de una sociedad barcelonesa más justa y cohesionada, como también una ciudad que necesita reflexionar sobre sus responsabilidades globales.

Una lectura reflexiva acerca del proceso desarrollado permite identificar algunas claves del éxito que podrían tenerse en cuenta en otros ámbitos o realidades en los que se pretenda incorporar el aprendizaje-servicio desde la tarea que realizan las entidades sociales:

- El punto de confluencia entre la educación para la justicia global y el aprendizaje-servicio, que comparten la idea de que la acción pedagógica es una práctica transformadora con dimensión política, orientada a la igualdad de oportunidades, que tiene como objetivo empoderar a las personas para ejercer una ciudadanía activa y responsable.

- El reconocimiento del potencial pedagógico y la capacidad de emprender proyectos de aprendizaje-servicio por parte de las entidades que trabajan en educación para la justicia global conjuntamente con centros educativos como punto de partida del programa.

- La voluntad de construcción conjunta que está en la base del trabajo entre entidades y centros educativos en las propuestas de aprendizaje-servicio, así como también en la esencia del trabajo colaborativo en el diseño e 
implementación del programa.

- La coherencia entre las diferentes líneas de trabajo, estrategias y acciones realizadas para dar respuesta y sentido a los objetivos del programa de manera completa y haciendo pedagogía hacia nuevas formas de hacer y pensar la educación para la justicia global.

- La búsqueda continua de la calidad como un criterio transversal que tiene que estar presente desde el acompañamiento a las entidades hasta el mismo diseño del programa, pasando por el desarrollo de proyectos y experiencias de aprendizaje-servicio en este ámbito.

- El equilibrio entre la construcción teórica y práctica del programa, procurando partir de las experiencias y el saber de las entidades a través del seguimiento y el análisis de proyectos para generar discurso sobre qué debe ser la educación para la justicia global.

- Una velocidad prudente pero a la vez constante que ha permitido crecer a un ritmo ascendente y sostenible, garantizando espacios periódicos para la reflexión sobre las líneas de trabajo y las acciones.

- La inversión de recursos a nivel personal y material para hacer posible un despliegue de calidad del programa.

A su vez, el proceso realizado hasta el momento también ha permitido detectar algunos retos y líneas de mejora que han de marcar la tarea a realizar a partir de aquí, y entre los que destacan:

- Consolidar los espacios de reflexión, intercambio de experiencias y aprendizajes, tanto a nivel individual como colectivo, como una forma de participar y co-crear soluciones y mejoras de las experiencias que se quieren desarrollar, así como trabajar hacia la mejora de la calidad de las propuestas. Estos espacios también han de permitir abordar nuevos debates actuales y desde diferentes disciplinas para hacer de esta propuesta una propuesta amplia y cada vez más rica en conocimientos y estrategias.

- Consolidar las convocatorias de subvenciones para que las entidades tengan recursos para desarrollar sus proyectos. Sería interesante adaptar las convocatorias de manera que se ayude tanto a propuestas más innovadoras e incipientes como a otras propuestas consolidadas que apuesten por mejoras cualitativas.

- La creación de líneas de trabajo específicas para llegar a nuevos públicos es un reto pendiente. Si tenemos en cuenta las potencialidades del ocio educativo y confiamos en una universidad abierta a la ciudad, será necesario plantear estrategias concretas para llegar a estos ámbitos. En ambos casos, la propuesta resulta realista a la vez que oportuna.

- La consideración, desde una perspectiva amplia, de incorporar a todos los agentes e implicarlos para

Monzó, M.; Rifà, J. y Rubio, L. (2018). Implementación y extensión del aprendizaje-servicio en las entidades de justicia global y cooperación internacional en la ciudad de Barcelona. RIDAS, Revista Iberoamericana de Aprendizaje Servicio, 6, 127-140. DOI10.1344/RIDAS2018.6.12 
que las acciones de todos los actores estén más coordinadas. En este sentido también, resulta importante consolidar las redes territoriales existentes y potenciar su autonomía fomentando la consolidación de los equipos de territorio.

- La implicación de otras áreas con entusiasmo que ven en el aprendizaje-servicio, y en este modelo de trabajo, una oportunidad de trabajo transversal para abrirse a nuevas metodologías y establecer proyectos entre diferentes ámbitos de manera coordinada.

- La incorporación y sistematización de la evaluación en los proyectos realizados por las entidades como propuesta de aprendizaje de los procesos y los resultados. En este sentido, la evaluación es también un instrumento de mejora de los proyectos.

- La difusión y comunicación como estrategias transversales para dar a conocer el programa, llegar a nuevos públicos, generar interés y compartir intereses con la ciudadanía.

\section{Conclusiones}

El trabajo que hemos presentado en este artículo permite poner de relieve que las experiencias de aprendizajeservicio suponen un cambio importante en la manera de trabajar de las entidades que se dedican a la educación para la justicia global. Además de avanzar hacia una educación transformadora, este tipo de proyectos impactan directamente en el tipo de relaciones entre los diferentes agentes sociales y educativos del territorio. Las entidades asumen nuevas tareas en su intervención educativa, toman un papel más proactivo en el diseño de proyectos y se construyen vínculos de confianza y reconocimiento mutuo. Por todo ello, parece interesante seguir apostando y avanzar en esta dirección en el futuro.

También cabe destacar, tal y como se ha puesto de manifiesto a lo largo del artículo, que el programa es el resultado de un proceso de trabajo cooperativo entre la universidad y la administración para el desarrollo de políticas públicas con voluntad transformadora, partiendo de la participación de los diferentes agentes implicados en un territorio. Se establece de esta manera, una nueva forma de trabajar entre la administración pública y la universidad, una relación colaborativa horizontal que rompe con los habituales roles de las instituciones.

El objetivo es contribuir a construir política pública para la ciudadanía contando con todos los agentes posibles de la ciudad. Para ello es imprescindible que cada institución, cada agente equitativamente y sin voluntad de protagonismo, aporte desde su saber y campo de acción. En este caso, siempre poniendo en el centro a las entidades, el valor de su tarea educativa y la potencialidad del aprendizaje-servicio en la misma

El aprendizaje-servicio resulta una propuesta educativa idónea para el 
trabajo de las entidades, no

únicamente las que trabajan en el ámbito de educación para la justicia global, que reconocen su papel educativo y buscan prácticas con mayor potencial pedagógico. Así mismo, estamos seguras que muchos de estos agentes pueden complementar $y$ contribuir a dar coherencia a la tarea que se realiza desde el ámbito de la educación para la justicia global.

Por todo ello, consideramos que la propuesta que aquí se ha presentado puede ser una vía de trabajo a explorar por las entidades y para otros departamentos de la Administración pública que quieran incorporar con éxito una estrategia educativa como el aprendizaje-servicio, así como también trabajar de manera coordinada y generar mayor coherencia con el resto de políticas del Ayuntamiento de modo que tanto los recursos invertidos como los resultados obtenidos tengan un mayor impacto en la ciudad.

\section{Referencias bibliográficas}

Ayuntamiento de Barcelona, Dirección de Justicia Global y Cooperación Internacional (2018). Pla Director de Cooperació per a la Justícia Global de Barcelona 2018-2021. Disponible en http://ajuntament.barcelona.cat/relacio nsinternacionalsicooperacio/sites/defaul t/files/pla_director_cooperacio_20182021_cat_v.web_0.pdf

Egea, A.; Barbeito, C.; Matamoros, M.; Massip, C. (2012). Competencias y EpD. La educación para el desarrollo en el currículum escolar desde la perspectiva de las competencias básicas. Barcelona: Edualter. Disponible en

https://www.uhu.es/IICIED/pdf/3_7_co mpete.pdf

Massip, C. (Coord.); Egea, A.; Barbeito, C. y Flores, M. (2018). Competències per transformar el món. Cap a una educació crítica i per a la justícia global a l'escola. Barcelona: Graó.

Nussbaum, M. C. (2010). Sin fines de lucro. Por qué la democracia necesita de las humanidades. Buenos Aires/Madrid: Katz Editores.

Puig, J.; Batlle, R.; Bosch, C. y Palos, J. (2007). Aprendizaje-servicio. Educar para la ciudadanía. Barcelona:

Octaedro.

Rubio, L. y Lucchetti. L. (2016). Aprenentatge servei, Pau, Drets Humans i Solidaritat. Noves propostes d'Educació per a la Justícia Global. Barcelona: Centro Promotor de Aprendizaje-Servicio, Fundació Jaume Bofill. Disponible en www.aprenentatgeservei.cat y en castellano https://apsvalors.wordpress.com/

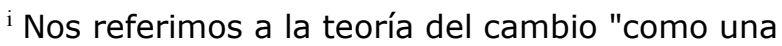
aproximación al análisis y la evaluación de la acción social que permite articular cómo y por qué una determinada actuación conduce a un cambio específico. Esta aproximación permite describir el conjunto de asunciones que explican la secuencia de pasos que conducen a la consecución de un objetivo, y las conexiones que existen entre las actividades concretas y el impacto real de una intervención o un programa a largo plazo. Así entendida, la Teoría del Cambio implica explorar el conjunto de creencias y asunciones sobre cómo se produce el cambio. Se trata de una aproximación muy utilizada en la evaluación de políticas sociales y también en el campo de la cooperación internacional al desarrollo" (Plan Anual de la
} 
Dirección de Justicia Global y Cooperación

Internacional, 2016).

Monzó, M.; Rifà, J. y Rubio, L. (2018). Implementación y extensión del aprendizaje-servicio en las entidades de justicia global y cooperación internacional en la ciudad de Barcelona. RIDAS, Revista Iberoamericana de Aprendizaje Servicio, 6, 127-140. DOI10.1344/RIDAS2018.6.12 